\title{
ESTOQUE DE CARBONO EM PLANTAÇÕES DE Pinus spp. EM DIFERENTES IDADES NO SUL DO ESTADO DO PARANÁ
}

\author{
Rafaelo Balbinot*, Álvaro Felipe Valério**, Carlos Roberto Sanquetta***, \\ Marcos Vinicius Winckler Caldeira****, Raul Silvestre***** \\ *Eng. Florestal, M.Sc., Depto. de Engenharia Ambiental, UNICENTRO - rbalbinot@yahoo.com.br \\ **Eng. Florestal, Mestrando em Ciências Florestais da UNICENTRO - alvarofvalerio@yahoo.com.br \\ ***Eng. Florestal, Dr., Depto. Ciências Florestais, UFPR - sanquetta@ufpr.br \\ ****Eng. Florestal, Dr., Depto. de Engenharia Florestal, UNICENTRO - caldeiramv@yahoo.com.br \\ *****Acadêmico de Engenharia Florestal, UNICENTRO - silvestrefloresta@yahoo.com.br \\ Recebido para publicação: 04/07/2007 - Aceito para publicação: 02/10/2007
}

\begin{abstract}
Resumo
Para a montagem de projetos florestais nos moldes do MDL, que atendam às exigências do mercado, será necessária a realização de estudos preliminares, testando-se tecnologias e gerando informações que possibilitem uma análise estratégica das potencialidades de uma região para as atividades desses projetos. O objetivo do trabalho foi analisar a dinâmica do estoque de carbono em plantações de Pinus spp. em diferentes classes de idade, entre os anos de 1993 e 2000, nos municípios de General Carneiro e Bituruna (PR), na região sul do estado do Paraná. Para isso, realizou-se um estudo do uso do solo com ênfase nas plantações florestais, por meio de imagens de satélite, seguindo os critérios e indicadores do Protocolo de Quioto. A região de estudo está contribuindo positivamente com a remoção de dióxido de carbono da atmosfera, pois foi constatado um aumento no estoque de carbono nas plantações florestais durante o período analisado. Esse último resultado não favorece o estabelecimento de projetos florestais de acordo com o Mecanismo de Desenvolvimento Limpo.

Palavras-chave: Fixação de carbono; MDL; reflorestamento; florestamento.
\end{abstract}

\section{Abstract}

Carbon stock in Pinus spp. plantations in different ages et southern region of the state of Paraná. The objective of this work was to quantify and analyze the carbon stock dynamism in Pinus spp. plantations at different age classes, between 1993 and 2000, in General Carneiro and Bituruna counties (PR), in southern region of Paraná. It was made, a study on the soil use with emphasis in forest plantations by satellite images and following criteria and index of Kyoto Protocol. The study region is positively contributing to remove carbon dioxide from the atmosphere, because an increase of carbon stock in forest plantations was verified during this analyzed time. This last outcome doesn't help the forest projects accomplishments according to the Clean Development Mechanism.

Keywords: Carbon sequestration; CDM; reforestation; aforestation.

\section{INTRODUÇÃO}

Atualmente, uma das questões de polêmica mundial é o aumento da concentração de dióxido de carbono $\left(\mathrm{CO}_{2}\right)$ na atmosfera e sua conseqüência sobre o efeito estufa (REZENDE, 2002). Embora a queima de combustíveis fósseis e a fabricação de cimento sejam as duas maiores fontes de emissão de $\mathrm{CO}_{2}$ para a atmosfera (BROWN; GILLESPIE; LUGO, 1989), vários estudos têm mostrado que os desmatamentos e outras mudanças no uso do solo também são significativas para o processo (DETWILER; HALL, 1988; DETWILER; HALL; BOGDONOFF, 1985).

Diante disso, a quantificação do estoque de carbono em florestas tem recebido mais atenção (BOSCOLO; BUONGIORNO, 1997; FEARNSIDE et al., 1999; KAUFFMAN; CUMMINGS; WARD, 1994; WOOMER; PALM, 1998), tanto pela possibilidade dessas áreas emitirem $\mathrm{CO}_{2}$ quanto pelo potencial de servirem como reservatórios de carbono e/ou sumidouros desse gás (CASTRO, 1996). Nesse sentido, as florestas são importantes para o equilíbrio do estoque de carbono global, pois armazenam em 
suas árvores e no solo mais carbono do que o existente atualmente na atmosfera. Se as florestas forem cortadas, a maior parte do carbono estocado será liberado para a atmosfera rapidamente, por meio de queimadas, ou mais lentamente, via decomposição (HOUGHTON, 1994). De acordo com Gardner; Mankin (1981), os ecossistemas florestais contêm cerca de 90\% da biomassa terrestre e cobrem aproximadamente $40 \%$ de sua superfície, apresentando elevada taxa de fixação de carbono, quando comparados com outras formações vegetais.

Permanecem incertezas sobre como diferentes usos da terra e atividades florestais afetam os estoques de carbono e as emissões de Gases de Efeito Estufa (GEE). O conhecimento do uso da terra é imprescindível para a elaboração de um cenário de referência para projetos de Mecanismo de Desenvolvimento Limpo (MDL), principalmente na modalidade florestamento/reflorestamento ${ }^{1}$.

O mercado para créditos de carbono está em plena expansão, e vários países estão conseguindo a aprovação de projetos para a venda desse tipo de serviço. Esse mercado representa uma alternativa de renda para o setor, podendo se estender até os pequenos produtores, por meio de associações (WATZLAWICK; MIRANDA; KOEHLER, 2005).

O objetivo do trabalho foi analisar a dinâmica do estoque de carbono em plantações de Pinus spp. em diferentes classes de idade, entre os anos de 1993 e 2000, nos municípios de General Carneiro e de Bituruna (PR), na região sul do estado do Paraná.

\section{MATERIAL E MÉTODOS}

\section{Área de estudo}

O município de General Carneiro encontra-se a $280 \mathrm{~km}$ da cidade de Curitiba, capital do Paraná, próximo à divisa com o estado de Santa Catarina, entre as coordenadas geográficas $26^{\circ} 25^{\prime} 39^{\prime}$ " de latitude

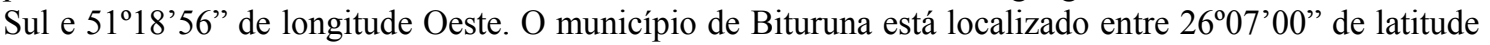
Sul e 51 $31^{\circ}$ '00" de longitude Oeste, com distância aproximada de 320 km de Curitiba (Figura 1).

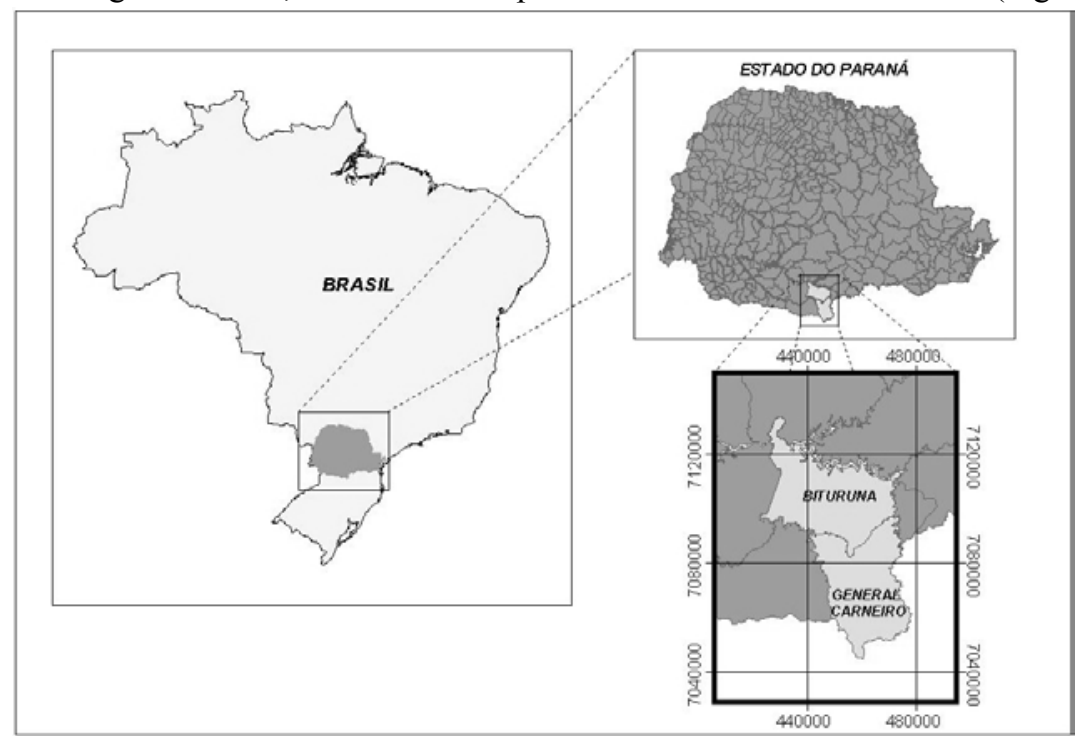

Figura 1. Localização da área de estudo.

Figure 1. Study area location.

O clima da região, conforme classificação de Köppen, é caracterizado como Subtropical Úmido Mesotérmico $(\mathrm{C} f b)$, com verões frescos e invernos com ocorrência de severas geadas. Não apresenta estações secas. A média das temperaturas dos meses mais quentes é inferior a $22{ }^{\circ} \mathrm{C}$, e a dos meses mais frios é superior a $18^{\circ} \mathrm{C}$ (PARANÁ, 1987).

\footnotetext{
${ }^{1}$ De acordo com o Protocolo de Quioto, o conceito de florestamento e reflorestamento diz respeito apenas à transformação de uma área sem cobertura florestal em uma área florestada, considerando tanto plantações comerciais quanto plantios para regeneração da vegetação original.
} 
Conforme Boletim de Pesquisa (1984), o substrato geológico da região é formado pelo derrame de Trapp da formação da Serra Geral. Os solos são predominantemente Neossolos Litólicos, Cambissolos e Argissolos, com ocorrências também de Organossolos. As características topográficas dividem-se em plana, ondulada e montanhosa, sendo a última de maior predominância (EMBRAPA, 1999).

\section{Levantamento do uso do solo com ênfase nos recursos florestais}

$\mathrm{Na}$ escolha das datas das imagens usadas como base para o estudo do uso do solo, levou-se em consideração o critério estabelecido pelo Protocolo de Quioto (PQ), que estabelece que as emissões de GEE devem ser reduzidas em 5,2\%, considerando os níveis observados em 1990. Logo, as considerações sobre um cenário de referência ${ }^{2}$ de projetos de MDL deve promover adicionalidade ${ }^{3}$ na fixação de carbono também com base nos valores observados em 1990, ou a data mais próxima que forneça as informações necessárias. A segunda data de referência do projeto foi determinada em função do Artigo $12.1^{4}$ do PQ, pelo qual os créditos de carbono podem ser contabilizados a partir do ano de 2000, para serem validados durante o primeiro período de compromisso do PQ de 2008 a 2012.

Para tanto, foram utilizadas imagens orbitais do satélite LANDSAT 5 para o ano de 1993, especificamente do dia 02/11/1993, e do satélite LANDSAT 7 para o ano de 2000, do dia 24/07/2000. A órbita-ponto para localização dessas cenas é 222/078. Para o processamento das imagens, foi utilizado o software ARCVIEW 3.1 by ERSI.

O meio utilizado foi o de classificação de imagens de satélite que recobrisse a área de interesse, isto é, os municípios de General Carneiro e Bituruna. Nesse contexto, os materiais que melhor esclareceram esse caso foram as imagens do satélite LANDSAT 5 e LANDSAT 7. Porém, não foi possível obter imagens de satélite satisfatórias para o ano de 1990, tampouco para os anos 1991 e 1992. Isso ocorreu devido a problemas nas imagens, que não estavam disponíveis em qualidade adequada, sempre apresentando nuvens ou problemas radiométricos. A imagem do ano de 1993 foi a mais próxima de 1990 que apresentava qualidade aceitável para o trabalho e estava disponível em arquivo digital. Há uma imagem da mesma área do ano de 1991, no entanto apresenta um defeito na sobreposição das bandas (originado por falha no sensor do satélite), que impossibilita estimativas exatas.

Para a realização da classificação das imagens de satélite, foi realizada coleta de pontos de controle, tendo como auxílio um aparelho de posicionamento global (Global Positon Sistem - GPS). Para aquisição desses pontos, levou-se em consideração o fácil reconhecimento no campo e na imagem, bem como que abrangessem todas as tipologias florestais a serem classificadas.

A digitalização da base cartográfica dos municípios, com caracterização da hidrografia, infraestrutura e demais detalhes, foi realizada com base em Cartas Topográficas da Diretoria de Serviço Geográfico do Exército Brasileiro e do Instituto Brasileiro de Geografia e Estatística (IBGE) em escala 1:50.000, sendo elas numeradas da seguinte forma: 2854, 2864, 2865 e 2877, recobrindo a área-alvo do estudo.

\section{Estimativas do estoque de biomassa e carbono}

As prognoses das produções das plantações de pinus foram feitas utilizando-se o simulador SISPINUS, desenvolvido pela EMBRAPA Florestas e pela UNIVERSIDADE FEDERAL DO PARANÁ (UFPR) (OLIVEIRA, 1995). O índice de sítio utilizado foi 21, que é adotado como médio para a região. O espaçamento de plantio utilizado nas análises foi $3 \times 2 \mathrm{~m}$, totalizando 1.600 árv.ha ${ }^{-1}$, com sobrevivência inicial de $95 \%$. A simulação considerou a realização de três desbastes, aos 8,12 e 16 anos, e corte raso aos 20 anos. O primeiro desbaste foi sistemático, pela remoção de uma linha de plantio em cada seis linhas, e seletivo, pela remoção de 304 árv.ha 1 , de acordo com características indesejáveis nessas árvores. O segundo desbaste foi somente seletivo, e foram retiradas 379 árv.ha $^{-1}$. O terceiro e último desbaste, também seletivo, promoveu a remoção de 228 árv. ha $^{-1}$. Posteriormente foram simuladas as produções para o corte raso aos 20 anos de idade.

\footnotetext{
${ }^{2}$ Cenário que quantifica e qualifica as emissões de gases de efeito estufa na ausência da atividade de projeto do MDL.

${ }^{3}$ Critério fundamental para que uma determinada atividade de projeto seja elegível ao MDL. Consiste na redução de emissões de gases de efeito estufa ou no aumento das remoções de $\mathrm{CO}_{2}$ de forma adicional ao que ocorreria na ausência de tal atividade.

${ }^{4}$ Artigo 12.1 do PQ: "Reduções certificadas de emissões obtidas durante o período do ano de 2000 até o inicio do primeiro período de compromisso podem ser utilizadas para auxiliar no cumprimento das responsabilidades relativas ao primeiro período de compromisso".
} 
A partir das estimativas de freqüência para cada classe diamétrica, foram aplicadas equações alométricas de biomassa seca para Pinus spp., desenvolvidas pelo Laboratório de Inventário Florestal da Universidade Federal do Paraná (UFPR/ECOPLAN, 2003), para seus diferentes compartimentos, sendo eles: peso de fuste (PF), peso de acículas (PAc), peso de galhos (PGv) e peso de raízes (PR), apresentados na tabela 1 .

Tabela 1. Equações de biomassa e coeficientes para Pinus spp. ajustadas pelo LIF/UFPR.

Table 1. Biomass equations and coefficients for Pinus spp. adjusted for LIF/UFPR.

\begin{tabular}{|c|c|c|c|c|c|}
\hline \multirow{2}{*}{ Compartimento } & \multirow{2}{*}{ Modelo } & \multicolumn{2}{|c|}{ Coeficientes do modelo } & \multirow{2}{*}{$\mathbf{R}^{2 \%}$} & \multirow{2}{*}{ Syx \% } \\
\hline & & $\mathbf{A}$ & b & & \\
\hline $\mathrm{PF}$ & \multirow{4}{*}{$\mathrm{a}\left(\mathrm{DAP}^{2} \mathrm{H}\right)^{\mathrm{b}}$} & 0,0595 & 0,9279 & 95,07 & 13,46 \\
\hline PAc & & 0,0012 & 1,0480 & 78,37 & 18,67 \\
\hline PGv & & 0,0001 & 1,3922 & 77,29 & 19,03 \\
\hline PR & & 0,4484 & 0,5619 & 63,64 & 32,98 \\
\hline
\end{tabular}

Após o cálculo da biomassa, esses valores foram convertidos para peso de carbono. Para isso, o valor da biomassa foi multiplicado pelo teor médio de carbono de cada compartimento das árvores de Pinus spp.: 0,454 para fuste, 0,408 para acículas, 0,446 para galhos vivos e 0,436 para raiz (UFPR/ECOPLAN, 2003).

\section{RESULTADOS E DISCUSSÕES}

\section{Uso do solo com ênfase nos recursos florestais}

As áreas com plantações florestais foram essencialmente das espécies Pinus taeda e Pinus elliottii. Observou-se que, no ano de 1993, havia uma baixíssima área com plantios de pinus com idade inferior a cinco anos, apenas 325 ha. Essa área reduzida em relação às áreas de plantios de outras idades pode ser conseqüência do fim dos incentivos fiscais em meados dos anos oitenta, e deve-se levar em conta que nessa época o futuro das florestas de pinus ainda era incerto. Desse modo, sem incentivos ou perspectivas que motivassem o investimento nesse setor, houve a formação de uma lacuna na continuidade dos plantios e conseqüentemente na oferta da madeira de pinus. Esse fato foi percebido pelas empresas de base florestal, que começaram a reagir plantando árvores com recursos próprios, fato que ocorreu mais intensamente no final da década de noventa (Tabela 2).

Tabela 2. Áreas com plantações florestais nos municípios de General Carneiro e de Bituruna, nos anos de 1993 e 2000 (em ha).

Table 2. Areas with forest plantations in the municipalities of General Carneiro and Bituruna (PR) in 1993 and 2000 (in ha).

\begin{tabular}{lcccccc}
\hline Classes de idade & $\mathbf{1 9 9 3}$ & \% do total & $\mathbf{2 0 0 0}$ & \% do total & $\begin{array}{c}\text { Diferença } \\
\mathbf{1 9 9 3}-\mathbf{2 0 0 0}\end{array}$ & $\begin{array}{c}\text { Mudança } \\
\text { \% }\end{array}$ \\
\hline$<5$ anos & 325,00 & 2,87 & $2.089,00$ & 12,10 & $1.764,00$ & 542,77 \\
$5-15$ anos & $3.077,00$ & 27,16 & $5.024,00$ & 29,10 & $1.947,00$ & 63,45 \\
$>15$ anos & $7.928,37$ & 69,97 & $10.152,00$ & 58,80 & $2.223,63$ & 28,05 \\
\hline Total & $11.330,37$ & 100,00 & $17.265,00$ & 100,00 & $5.934,63$ & 52,38 \\
\hline
\end{tabular}

O efeito dessa iniciativa já pôde ser constatado no ano de 2000, quando existiam na área dos dois municípios aproximadamente 2.089 ha de pinus com idades inferiores a 5 anos, um aumento de $542 \%$. Analisando a dinâmica das plantações de pinus, das classes 5 a 15 anos e com mais de 15 anos, constatouse que nos dois casos houve aumento da área plantada da ordem de 63 e $28 \%$, respectivamente. Esse aumento representa uma área de aproximadamente 4.171 ha.

De modo geral, o aumento médio foi superior a 52\%, porém ainda existe uma grande disparidade entre as áreas com florestas acima de 15 anos, que representam 58\% do total, e as florestas com 5 a 15 anos, que somam $29,1 \%$ do total. As florestas com menos de 5 anos representam apenas $12,1 \%$ do total 
da cobertura de plantações florestais. Ou seja, o risco de falta de madeira na região de estudo é um fato, principalmente se considerarmos a tendência de aumento do consumo de produtos de base florestal.

\section{Estoque de biomassa e carbono}

$\mathrm{O}$ fuste representou o maior percentual de biomassa e carbono, com tendência a aumentar à medida que a árvore cresce. No caso deste trabalho, o carbono presente na biomassa do fuste totalizou mais de $71 \mathrm{Mg} . \mathrm{C} . \mathrm{ha}^{-1}$, representando cerca de $70 \%$ de todo o carbono fixado.

Outro resultado marcante é a importância das raízes no total do carbono fixado, chegando a representar no caso das plantações com menos de 5 anos, 35\% do carbono fixado, 21\% para plantações entre 5 e 15 anos e 13\% naquelas com mais de 15 anos. Esses resultados de biomassa e carbono encontrados nas raízes de plantações florestais mostram que não se pode prescindir da quantificação do carbono presente no sistema radicial, principalmente se o objetivo for apresentar estimativas corretas sobre a capacidade das florestas de fixar carbono atmosférico e, conseqüentemente, gerar créditos comercializáveis.

Na tabela 3, são apresentados os valores estimados de biomassa e carbono por hectare para fuste, acículas, galhos vivos e raízes. Esses valores, apresentados para cada classe de idade de pinus, são valores líquidos do total de carbono fixado pela floresta, ou seja, descontadas as remoções provocadas por ocasião da aplicação dos desbastes.

Tabela 3. Biomassa e carbono por hectare nos diferentes compartimentos das árvores de pinus.

Table 3. Biomass and carbon per hectare in different compartments of pinus trees.

\begin{tabular}{|c|c|c|c|c|c|}
\hline \multirow{2}{*}{ Idades (anos) } & \multicolumn{5}{|c|}{ Biomassa $\left(\right.$ Mg.ha $\left.^{-1}\right)$} \\
\hline & Fuste & Acículas & Galhos vivos & Raízes & Total \\
\hline$<5$ & 33,40 & 1,73 & 1,57 & 20,23 & 56,93 \\
\hline $5-15$ & 113,02 & 7,18 & 11,66 & 36,05 & 167,91 \\
\hline$>15$ & 156,69 & 11,72 & 30,15 & 29,95 & 228,41 \\
\hline \multirow{2}{*}{ Idades (anos) } & \multicolumn{5}{|c|}{ Carbono $\left({\left.\mathrm{Mg} . \mathrm{ha}^{-1}\right)}^{-1}\right.$} \\
\hline & Fuste & Acículas & Galhos vivos & Raízes & Total \\
\hline$<5$ & 15,17 & 0,77 & 0,68 & 8,95 & 25,57 \\
\hline $5-15$ & 51,33 & 3,20 & 5,06 & 15,96 & 75,55 \\
\hline$>15$ & 71,13 & 5,23 & 13,08 & 13,26 & 102,70 \\
\hline
\end{tabular}

O total de carbono fixado pelas plantações de pinus com mais de 15 anos, aproximadamente 102 Mg.C.ha ${ }^{-1}$, foi próximo do encontrado por Schumacher (2002): 114,84 Mg.C.ha ${ }^{-1}$ e 133,39 Mg.C.ha-1 em plantações de Pinus taeda com 15 e 20 anos, respectivamente, no Rio Grande do Sul.

Sette Junior; Nakajima; Geromini (2006), estudando a captura de carbono orgânico em povoamentos de Pinus taeda L. aos 5, 12, 14, 18 e 25 anos, na região de Rio Negrinho (SC), encontraram a maior quantidade de carbono orgânico para o povoamento de 18 anos, com 109,9 Mg.C.ha ${ }^{-1}$, sendo essa quantidade fixada muito próxima à encontrada neste estudo para os povoamentos com idade superior a 15 anos.

Balbinot et al. (2003), em inventário do carbono orgânico em um plantio de Pinus taeda com 5 anos de idade no Rio Grande do Sul, encontrou um volume de $18,8 \mathrm{Mg} \cdot \mathrm{ha}^{-1}$ de carbono estocado, inferior ao encontrado neste estudo com o gênero Pinus $\left(25,57 \mathrm{Mg}^{-h a^{-1}}\right)$.

A partir das quantificações do total de carbono fixado por hectare, para as plantações de pinus da região, foi gerada a tabela 4 , que apresenta a evolução do estoque de carbono no período considerado (1993-2000), de acordo com a área calculada para cada classe de idade.

Houve aumento das plantações de pinus na região, ocorrendo, conseqüentemente, um aumento do total de carbono estocado no período de 1993 a 2000. Esse aumento foi de $420.000 \mathrm{Mg}$.C (ou aproximadamente $1.500 .000 \mathrm{Mg} / \mathrm{CO}_{2}$ ). Certamente um valor significativo, que está concentrado nas áreas com plantações com idade superior a 15 anos.

É importante ressaltar que esse aumento ocorreu de forma independente da presença de projetos florestais incentivados pelo MDL. Devido a isso, comparativamente a uma região onde tenha ocorrido uma redução no estoque de carbono (igual à redução na área de uso do solo com plantações florestais), a região dos municípios de Bituruna e General Carneiro será menos atrativa para a implantação de projetos de florestamento e reflorestamento que visem geração de créditos de carbono, pois apresentou, no período considerado, aumento nos estoques de carbono em plantações florestais. A reversão desse quadro só 
acontecerá caso haja uma suplementação de área plantada, e, além disso, não sejam instaladas novas indústrias consumidoras de madeira nesse período.

Tabela 4. Estoque de carbono dos plantios de Pinus spp. nos municípios de General Carneiro e Bituruna em 1993 e 2000.

Table 4. Carbon stock in Pinus spp. plantations in the counties of General Carneiro and Bituruna in 1993 and 2000.

\begin{tabular}{lcccccc}
\hline \multirow{2}{*}{ Classes de idade } & \multicolumn{7}{c}{ Carbono $\left(\mathbf{M g . h a} \mathbf{- 1}^{-\mathbf{1}}\right.$} \\
\cline { 2 - 7 } & $\begin{array}{c}\text { Ano de 1993 } \\
\mathbf{( M g )}\end{array}$ & $\mathbf{\%}$ do total & $\begin{array}{c}\text { Ano de 2000 } \\
\mathbf{( M g )}\end{array}$ & \% do total & $\begin{array}{c}\text { Diferença } \\
\mathbf{1 9 9 3}-\mathbf{2 0 0 0}\end{array}$ & \% \\
\hline$<5$ anos & 8.310 & 0,79 & 53.415 & 3,62 & 45.105 & 542,78 \\
$5 \geq 15$ anos & 232.467 & 22,03 & 379.563 & 25,72 & 147.096 & 63,45 \\
$>15$ anos & 814.243 & 77,19 & 1.042 .610 & 70,66 & 228.367 & 28,05 \\
\hline Total & 1.055 .020 & 100 & 1.475 .588 & 100 & 420.569 & 39,86 \\
\hline
\end{tabular}

A partir das estimativas de carbono fixado por hectare em plantações de pinus da tabela 3, foram estimadas as quantidades de $\mathrm{CO}_{2}$ que seriam removidas da atmosfera, conforme é apresentado na tabela 5 . A obtenção desse valor foi feita por meio da multiplicação do peso atômico do carbono por 3,6667, que é o fator de conversão dos pesos atômicos da molécula de $\mathrm{C}$ (peso atômico 12) para a de $\mathrm{CO}_{2}$ (peso atômico 44), pois a valoração do serviço da floresta de fixar carbono, e que deve servir de base para os cálculos do fluxo de caixa para um possível projeto, é contabilizada em toneladas de $\mathrm{CO}_{2}$.

Tabela 5. $\mathrm{CO}_{2}$ removido da atmosfera, por hectare, em plantações de pinus em diferentes compartimentos das árvores e idades.

Table 5. $\mathrm{CO}_{2}$ removed from the atmosphere per hectare in pinus plantations on different compartments of trees and ages.

\begin{tabular}{lccccc}
\hline \multirow{2}{*}{ Idades (anos) } & \multicolumn{5}{c}{ Dióxido de carbono $\left(\mathbf{M g . h a}^{-\mathbf{1}}\right)$} \\
\cline { 2 - 6 } & Fuste & Acículas & Galhos vivos & Raízes & Total \\
\hline$<5$ & 55,63 & 2,82 & 2,49 & 32,82 & $\mathbf{9 3 , 7 7}$ \\
$5 \geq 15$ & 188,23 & 11,73 & 18,55 & 52,52 & $\mathbf{2 7 7 , 0 4}$ \\
$>15$ & 260,83 & 19,18 & 47,96 & 48,62 & $\mathbf{3 7 6 , 6 0}$ \\
\hline
\end{tabular}

O interesse dos produtores rurais em implantar florestas é bastante significativo, especialmente para áreas e solos marginais sem aptidão para a agricultura. Assim, o produtor viabiliza economicamente algumas áreas não utilizadas. O reflorestamento será uma "poupança verde", à qual poderá recorrer de acordo com sua necessidade, além de receber, durante o período do projeto, os valores referentes à remoção de $\mathrm{CO}_{2}$ atmosférico por sua floresta. Considerando-se a média de $376,6 \mathrm{Mg}$ de $\mathrm{CO}_{2}$ removidas da atmosfera por plantações de pinus com mais 15 anos, e um valor de comercialização de US\$ 4.00 sob uma cotação do dólar de $\mathrm{R} \$ 2,20$, ter-se-ia uma remuneração de aproximadamente $\mathrm{R} \$ 3.314,08$.

Os resultados referentes à análise de uso do solo entre 1993 e 2000 mostraram que houve um aumento das plantações florestais de pinus. $\mathrm{O}$ aumento de área plantada promoveu, conseqüentemente, um aumento do estoque de carbono, pois a região pertencente aos dois municípios em estudo está contribuindo positivamente para a remoção de $\mathrm{CO}_{2}$ atmosférico.

Essa linha de base, que apresentou saldo positivo na soma das mudanças nos estoques de carbono nos reservatórios (1993-2000), é desfavorável ao intuito de implantar projetos de florestas fixadoras de carbono, isso porque a Decisão 19 da $9^{\text {a }}$ Conferência das Partes (COP 9), parágrafo 20 (c), indica que, para o cálculo da linha de base, devem ser consideradas as políticas e circunstâncias nacionais e/ou setoriais relevantes, tais como uso da terra histórico e tendências econômicas.

\section{CONCLUSÕES}

Constatou-se um aumento significativo das áreas com plantações florestais no período analisado (1993-2000), que promoveu, conseqüentemente, um acréscimo nos estoques de carbono. Assim, a região de estudo está contribuindo positivamente para a remoção de dióxido de carbono da atmosfera. 
Em conseqüência disso, se compararmos a área de estudo a uma região onde tenha ocorrido uma redução nos estoques de carbono, ela será considerada menos atrativa para a implantação de projetos de florestamento e reflorestamento que visem geração de créditos de carbono nos moldes do MDL.

Apesar de não objetivarmos avaliar o uso de imagens de satélite, pudemos concluir que a tecnologia de classificação de imagens do satélite LANDSAT, utilizada para analisar a evolução do uso da terra e dos estoques de carbono da vegetação florestal, à luz dos conceitos do PQ, mostrou-se viável, além de ter custo relativamente baixo, especialmente para estudos estratégicos como esse, com grande área de cobertura.

\section{REFERÊNCIAS}

BALBINOT, R.; SCHUMACHER, M. V.; WATZLAWICK, L. F.; SANQUETTA, C. R. Inventário do carbono orgânico em um plantio de Pinus taeda aos 5 anos de idade no Rio Grande do Sul. Revista de Ciências Exatas e Naturais, Guarapuava, v.5, n. 1, p 59-68, jan./jun., 2003.

BOSCOLO, M.; BUONGIORNO, J. Managing a tropical rainforest for timber, carbon storage and tree diversity. Commonwealth Forestry Review, Oxford, v. 76, n. 4, p. 246-254, 1997.

BROWN, S.; GILLESPIE, A. J. R.; LUGO, A. Biomass estimation methods for tropical forests with applications to forest inventory data. Forest Science, Bethesda, v. 35, n. 4, p. 881-902, 1989.

CASTELA, P. R. (Coord.). Conservação do bioma floresta com Araucária: relatório final. Curitiba: FUPEF, 2001. v.1/2.

CASTRO, E. A. Biomass, nutrient pools and response to fire in the Brazilian Cerrado. $128 \mathrm{f}$. MS Thesis - Oregon State University, Oregon, 1996.

DETWILER, R. P.; HALL, C. A. Tropical forest and the global cycles. Science, S.Washington, DC, v. 239, p. 42-47, 1988.

DETWILER, R. P.; HALL, C. A.; BOGDONOFF, P. Land use change and carbon exchange in the tropics: II. Estimates for the entire region. Environmental Management, New York, v. 9, p. 335-344, 1985.

EMBRAPA - Centro Nacional de Pesquisa de solos. Sistema brasileiro de classificação de solos. Brasília, DF: EMBRAPA-SPI; Rio de Janeiro: EMBRAPA-CNPS, 1999. 412 p.

FEARNSIDE, P. M.; GRAÇA, P. M. L. A.; LEAL FILHO, N.; RODRIGUES, F. J. A.; ROBINSON, J. M. Tropical forest burning in Brazilian Amazonia: measurement of biomass loading, burning efficiency and charcoal formation at Altamira, Pará. Forest Ecology and Management, Amsterdam, v. 123, n.1, p. 65-79, 1999.

GARDNER, R. H.; MANKIN, J. B. Analysis of biomass allocation in forest ecosystems of the IBP. In: REICHLE, P. D. Dynamic properties of forest ecosystems. Cambridge: Cambridge University Press. 1981. p. 451-497.

HOUGHTON, R. A. As florestas e o ciclo de carbono global: armazenamento e emissões atuais. In: SEMINÁRIO EMISSÃO X SEQÜESTRO DE $\mathrm{CO}_{2}$ - UMA NOVA OPORTUNIDADE DE NEGÓCIOS PARA O BRASIL, 1994, Rio de Janeiro. Anais... Rio de Janeiro: Companhia Vale do Rio Doce, 1994. p. $38-76$.

INSTITUTO BRASILEIRO DE GEOGRAFIA E ESTATÍSTICA (IBGE). Vegetação e geografia do Brasil - Região Sul. Rio de Janeiro: IBGE, 1992. v. 2.

KAUFFMAN, J. B.; CUMMINGS, D. L.; WARD, D. E. Relationships of fire, biomass and nutrient dynamics along vegetation gradient in the Brazilian cerrado. Journal of Ecology, London, v. 82, p. 519$531,1994$.

LARCHER, W. Ecofisiologia vegetal. São Paulo: Ed. Pedagógica e Universitária, 2000. 320 p. 
EMBRAPA - Levantamento de reconhecimento dos solos do estado do Paraná. Londrina: EMBRAPA / SUDESUL, 1984. $791 \mathrm{p}$.

OLIVEIRA, E. B. Um sistema computadorizado de prognose do crescimento e produção de Pinus taeda L., com critérios quantitativos para avaliação técnica e econômica de regimes de manejo. 134 f. (Tese - Doutorado em Ciências Florestais) - Setor de Ciências Agrárias, Universidade Federal do Paraná, Curitiba, 1995.

PARANÁ. Secretária de Estado de Agricultura e Abastecimento, Instituto de Terras, Cartografia e Florestas. Atlas do estado do Paraná. Curitiba, 1987. 73 p.

REZENDE, A. V. Diversidade, estrutura, dinâmica e prognose do crescimento de um cerrado sensu stricto submetido a diferentes distúrbios por desmatamento. 229 f. Tese (Doutorado em Manejo Florestal) - Setor de Ciências Agrárias, Universidade Federal do Paraná, Curitiba, 2002.

SCHUMACHER, M. V. Estoque de carbono em florestas de Pinus taeda L. E Acacia mearnsii De Wild plantadas no estado do Rio Grande do Sul-Brasil. In: SANQUETTA, C. R.; et al. (Eds ). As Florestas e o Carbono. Curitiba, 2002. p. 141-152.

SETTE JR, C. R.; NAKAJIMA, N. Y.; GEROMINI, M. P. Captura de carbono orgânico em povoamentos de Pinus taeda L. na região de Rio Negrinho, SC. Revista Floresta, Curitiba, vol. 36, n. 1, p. 33-44, jan/abr., 2006.

UFPR / ECOPLAN - Universidade Federal do Paraná e Instituto Ecoplan. Estudo de viabilidade para implantação de florestas fixadoras de carbono: estudo de caso no sul do estado do Paraná. Curitiba, $2003.93 \mathrm{p}$.

WATZLAWICK, L. F.; MIRANDA, G. M.; KOEHLER, H. S. Quantificação e valoração econômica de biomassa e carbono. Atualidades Florestais e Ambientais, Irati, 179 p, 2005.

WOOMER, P. L.; PALM, C. A. An approach to estimating carbon stocks in tropical forests and associated land uses. Commonwealth Forestry Review, Oxford, v. 77, n. 3, p. 181-190, 1998. 